\title{
Construction and Tests
}

\section{of an Injection Electron Gun for a $6.7 \mathrm{GHz}$ Monotron}

\author{
J. J. Barroso, J. O. Rossi, P. J. Castro, J. A. N. Gonçalves, and E. Del Bosco \\ Associated Plasma Laboratory, National Institute for Space Research - INPE \\ 12201-970 São José dos Campos, SP, Brazil
}

\begin{abstract}
We describe the construction and emission tests of an electron gun used to accelerate and inject a hollow electron beam into a cylindrical cavity to produce high-power microwaves at $6.7 \mathrm{GHz}$, a frequency suitable for communications, medical and industrial applications. In addition to the monotron components, a hard-tube pulser with three tetrodes in parallel is also discussed, able to generate negative high voltages up to 30 $\mathrm{kV} / 20 \mathrm{~A}$ from a positive DC charging power supply without using a floating switch.
\end{abstract}

Index Terms - electron gun, microwave generation, monotron, oxide cathodes, high-voltage power supply.

\section{INTRODUCTION}

Consisting of a cylindrical cavity driven by a rectilinear electron beam, the monotron is the simplest microwave generator, whose principle of operation relies on transit-time effects experienced by the electron beam propagating across a resonant cavity. Here we describe an electron gun used to produce and inject a hollow electron into a cylindrical cavity to generate high-power microwaves at $6.7 \mathrm{GHz}$. Currently under development at our laboratory, this monotron [1] currently operates with a beam of about $3.0 \mathrm{~A}$ at $10 \mathrm{kV}$ as discussed throughout the paper in connection with the operating characteristics of the electron gun

\section{GUN CONSTRUCTION}

Shown in Fig. 1 is the complete layout of the $6.7 \mathrm{GHz}$ monotron. The assembly includes an injection electron gun, a resonant cavity coupled to the TM-mode output waveguide through a coupling hole. The cathode arrangement is devised to bend the equipotential surfaces so as to confine and focus the beam even in the presence of space-charge space forces as the electrons move away from the emitting surface. In the lower panel, it is shown the electron beam bunched in the steady-sate regime, in which DC beam power is converted into electromagnetic power at a typical 20 percent conversion efficiency. Without divergence, the beam is injected into the cavity through an annular slot.

Since the cathode should operate at temperatures close to $1000{ }^{\circ} \mathrm{C}$, the choice of materials for its construction is limited. As for the requirements of mechanical strength and low vapor pressure at high temperature, materials of low emissivity and poor thermal properties are needed to reduce heating power losses. The most suitable materials with such properties are the refractory metals and their alloys. Of these, molybdenum was selected for the cavity's entrance plate onto which, to allow passage of the electron beam, a pair of symmetric slots was drilled as viewed on the left side in Fig. 2. On the right and closing a ceramic pot, it is shown the circular cathode plate made from nickel on which the electron emission coating is applied. Housing the heater filament inside, the ceramic pot is held by a circular disk of titanium, which has a thermal conductivity of $22 \mathrm{~W} / \mathrm{m} / \mathrm{K}$, a low value when either compared with molybdenum $(138 \mathrm{~W} / \mathrm{m} / \mathrm{K})$ or tungsten $(173$ $\mathrm{W} / \mathrm{m} / \mathrm{K})$. The supporting disk stays fastened to three circular stainless steels rods directly attached to the vacuum flange.

The heater is made of a 0.5 -mm-diameter pure tungsten wire tightly wound, with the resulting helix bent to take the shape of a toroidal coil (Fig. 3) housed on the back of the cathode disk to heat the emitting region radiatively.
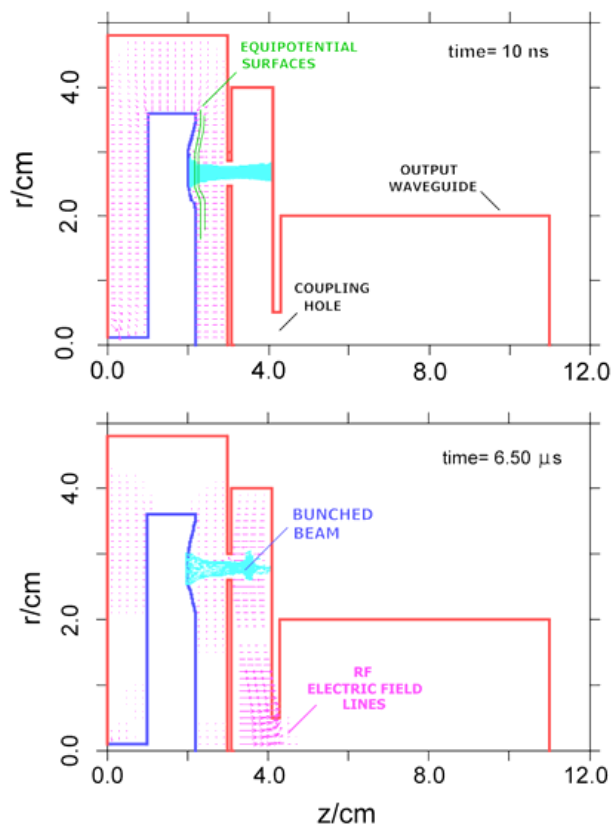

Fig. 1. Monotron configuration showing the beam electrons at early times (upper panel) and in the steady state (lower panel) of the RF field-beam interaction 


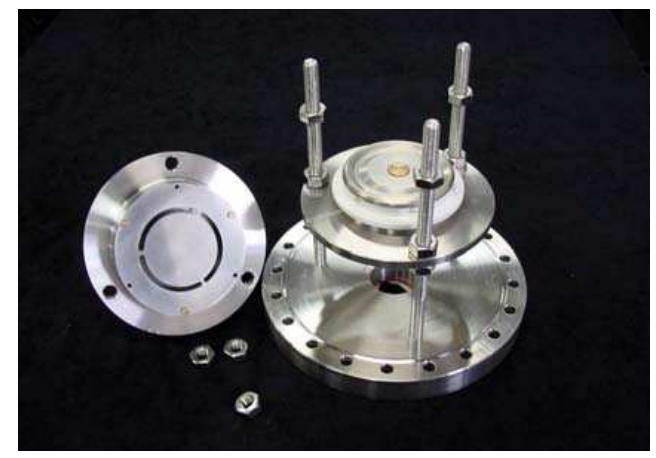

Fig. 2. Front plate of the circular cavity and gun assembly.

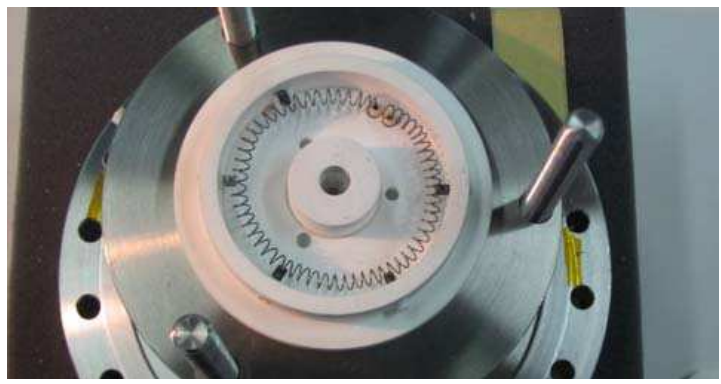

Fig. 3. Heating tungsten filament housed in the ceramic pot.

\section{High-Voltage POWER Supply}

The $6.7 \mathrm{GHz}$ monotron had been operating at a pulse current of about $3.0 \mathrm{~A}$, duration of $20 \mu$ s with a repetition rate $50 \mathrm{~Hz}$, at a $10 \mathrm{kV}$ beam voltage produced by a pulser configuration circuit [2] with a floating high voltage on-off switch shown in Fig. 4. To be able to generate beams with still higher current, we have developed a new configuration of power supply with three tetrodes in parallel (Fig. 5). In this scheme, the high voltage switch and its grid power supplies are near the ground, which eases the maintenance and the pulser construction. In fact, the on-off switching uses a power MOSFET in series with the cathodes of three tetrodes of $8 \mathrm{~A}$ maximum plate current each connected in parallel. This type of circuit configuration is very convenient because the tubes withstand the major fraction of the blocking voltage as IGBTs operate at maximum voltages up to $1.5 \mathrm{kV}$ and they do not require high power gate drivers. As the cathode current is switched directly without any protecting resistor and the tube has low impedance during the switching, this leads to a very short pulse rise time. When the anode potential has dropped below the screen grid voltage during a fault, the tube current flows mostly to the grids instead to the cathode causing a drop of the grid voltages, which reduces the cathode current to the value required by the load. A voltage pulse produced by this power supply is illustrated in Fig. 6.

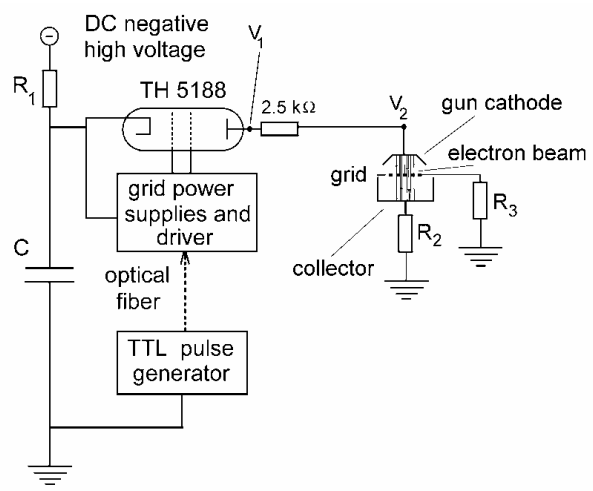

Fig. 4 A circuit pulser using a floating high voltage switch.

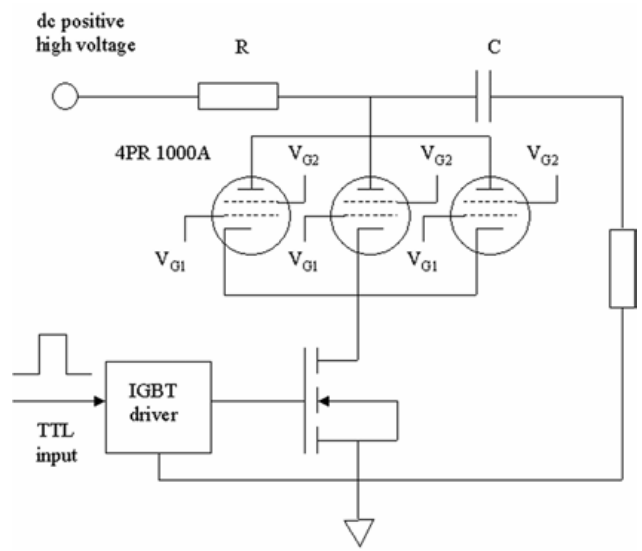

Fig. 5. IGBT-controlled high-voltage power supply with three tetrodes in parallel.

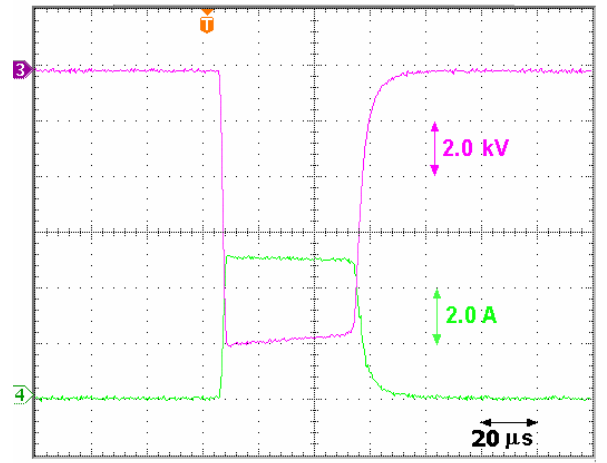

Fig. 6. From the power supply in Fig. 5, a voltage pulse (red trace) across a $2.0 \mathrm{k} \Omega$ load giving a $5.0 \mathrm{~A}$ current pulse (green trace). 


\section{THERMAL TEST}

To produce $30 \mathrm{~kW}$ output power at $10 \mathrm{kV}$, a $20 \mathrm{~A}$ beam current is required on the basis of a $15 \%$ experimental conversion efficiency. At this requirement, an emitter which could provide at least $3.0 \mathrm{~A} / \mathrm{cm}^{2}$ current density is desirable. Furthermore, because of the large size of the cathode disk $\left(\sim 41.0 \mathrm{~cm}^{2}\right)$, the required emission should be available at as low a temperature as possible to minimize the necessary heater power and to reduce radiative heat of surrounding components. While requiring a rugged long-life emitter having good emission properties at a low temperature, an oxide cathode is a reliable choice for the thermal electron emitter. The selected material for cathode coating is the triple carbonate $(\mathrm{Ba}, \mathrm{Sr}, \mathrm{Ca}) \mathrm{CO}_{3}$, which is still widely used in picture tubes and similar applications. Developed at our laboratory [3], the coating mixture used differs from the standard commercial composition [4] by having a higher percentage of absolute ethyl alcohol $(22.9 \%$ instead the usual percentage of $6.6 \%$ ) and a lower fraction of nitrocellulose $(2.9 \%$ instead of $12.2 \%)$. Such alterations make easier the application by spray in addition to reducing the drying time of the coating. The so prepared suspension was sprayed onto the cathode annular band and dried at $100{ }^{\circ} \mathrm{C}$. The mechanism of the oxide cathode operation relies on the release of free barium, within the bulk of the coating during the activation process, which then migrates to the surface to form a monoatomic film of barium.

The cathode activation process involves three steps: (1) removal of the nitrocellulose binder (normally occurring at around $350{ }^{\circ} \mathrm{C}$ ), (2) conversion of the carbonates into oxides $\left(\mathrm{SrCO}_{3} \rightarrow \mathrm{Sr}+\mathrm{CO}_{2}\right.$ and $\mathrm{CaCO}_{3} \rightarrow \mathrm{CaO}+\mathrm{CO}_{2}$, at about 620 ${ }^{\circ} \mathrm{C}$ ), and (3) partial reduction of the oxides $\left(\mathrm{BaCO}_{3}\right.$ $\rightarrow \mathrm{BaO}+\mathrm{CO}_{2}$, around $900{ }^{\circ} \mathrm{C}$ ) at the metal-coating interface to produce free barium throughout the coating by diffusion of $\mathrm{Ba}+$ and $\mathrm{Sr}+$. After conversion of the carbonate into oxide has been completed, activation proper is achieved by raising the cathode temperature to $\sim 1000{ }^{\circ} \mathrm{C}$ for a few minutes.

To wok out a suitable activation schedule, the full monotron system was mounted and measurements were taken of the cathode temperature as function of the heater power. To this end, calibrated K-type thermocouples (Fig. 7) and digital meters were used to measure the temperatures at the annular emitting band and at the center of the cathode disk, respectively indicated by $T_{1}$ and $T_{2}$ in Fig. 8. Input heater power was supplied by an AC source and pressure was kept below $10^{-5}$ torr by operating a vacuum diffusion pump.

The thermal behavior of the cathode is shown in Fig. 9, which indicates that to reach the operating temperature of 900 ${ }^{\circ} \mathrm{C}$ an amount of $500 \mathrm{~W}$ heater power is required.

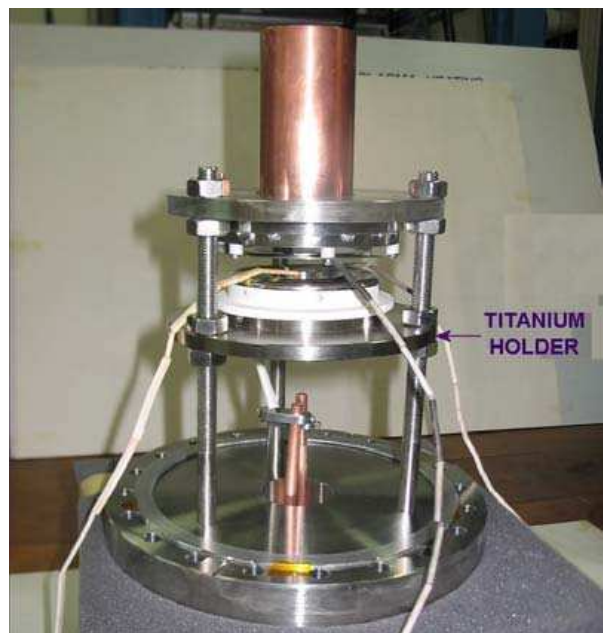

Fig. 7. Full monotron assembly with measuring thermocouples.

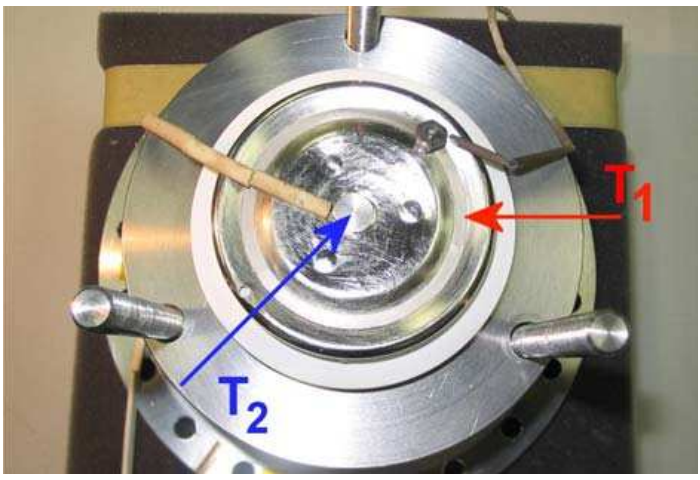

Fig. 8. Top view of the cathode nickel plate.

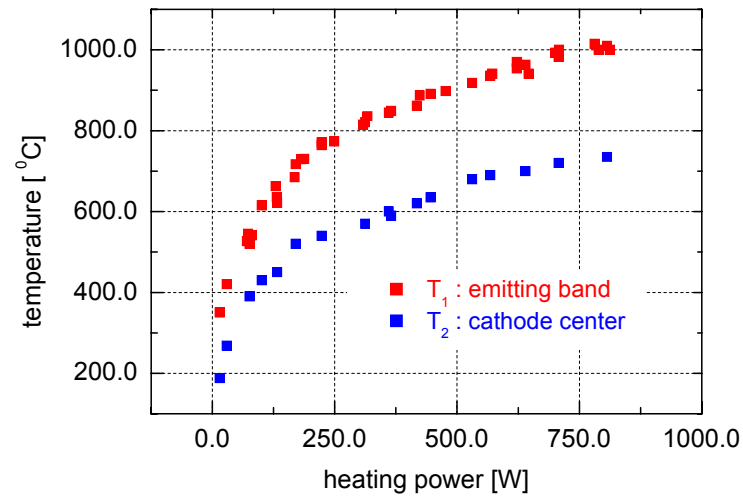

Fig. 9. Thermal behavior of the cathode showing temperature dependence upon heating input power. 


\section{EMISSION TEST}

Thermal electron current density is adequately described by the Richardson-Dushman equation [4]

$$
J_{s}=A T^{2} \exp \left(-\frac{\phi}{k T}\right)
$$

where $A=1.20 \times 10^{6} \mathrm{~A} / \mathrm{m}^{2} / \mathrm{K}^{2}$ is a constant, $\phi$ the emitter true work function, and $k=1.38 \times 10^{-23} \mathrm{~J} / \mathrm{K}=8.6 \times 10^{-5} \mathrm{eV} / \mathrm{K}$ the Boltzmann's constant. Assuming the work function $\phi$ varies linearly with temperature, $\phi=\phi_{0}+\alpha T$, gives the coefficient $A_{0}=$ $A \exp (-\alpha / k)$. In practice, the value of $\alpha$ is of the same order of $k$, resulting in a coefficient $A_{0}=40 \mathrm{~A} / \mathrm{cm}^{2} / \mathrm{K}^{2}$ for most metals; taking the same $\phi$ for all regions of the emitting surface, the experimental value of $A_{0}$ lies between 30 and $70 \mathrm{~A} / \mathrm{cm}^{2} / \mathrm{K}^{2}$. A significant aspect of (1) is the exponential variation of $J_{s}$ with temperature, in that the variation caused by the quadratic term $T^{2}$ is negligible by comparison. In fact, the relative variation in current density $\Delta J / J$ arising from $T^{2}$ amounts to $2 \Delta T / T$, while the exponential term yields $\Delta J / J=(\phi / k T) \Delta T / T$. For instance, taking $\Delta T / T=1 \%$ and $\phi=2.0 \mathrm{eV}$ gives $2 \%$ and $48 \%$, respectively. Usually referred to as temperature-limited operation, this regime corresponds to running the emitter above the saturation voltage, a condition in which the anode attracts all the electrons emitted from the cathode and a further increase in voltage does not raise the current as much as a small increase in temperature does.

Preliminary emission measurements are shown in Fig. 10 with experimental data fitted by (1), yielding the RichardsonDushman coefficient $A_{0}=3.5 \mathrm{~A} / \mathrm{cm}^{2} / \mathrm{K}^{2}$ and the work function $\phi=1.8 \mathrm{eV}$. Because of the exponential dependence of the current density $J_{s}$ on the work function, a somewhat higher value $\phi=2.0 \mathrm{eV}$ would give the six-fold higher coefficient $A_{0}=22.1 \mathrm{~A} / \mathrm{cm}^{2} / \mathrm{K}^{2}$ but with the corresponding fitting curve almost indistinguishable from that shown in Fig. 10. Upon comparison, the pressed Philips dispenser cathode [4] has the emission parameters $A_{0}=2.4 \mathrm{~A} / \mathrm{cm}^{2} / \mathrm{K}^{2}$ and $\phi=1.7 \mathrm{eV}$. Intended for mass production applications, that cathode produces a typical current density of $2.5 \mathrm{~A} / \mathrm{cm}^{2}$ at the operating temperature of $1400 \mathrm{~K}$, while our emitter has given a current density of $0.6 \mathrm{~A} / \mathrm{cm}^{2}$ at $1300 \mathrm{~K}$.

Typical experimental voltage and current pulses are displayed in Fig. 11. Comparing the rectangular current pulse in Fig. 6 with the emission current pulse, the exponential decay occurring on the central part of the pulse (Fig.11) is due to stray capacitances that arise from the presence of metallic elements surrounding the cathode and the cavity entrance plate.

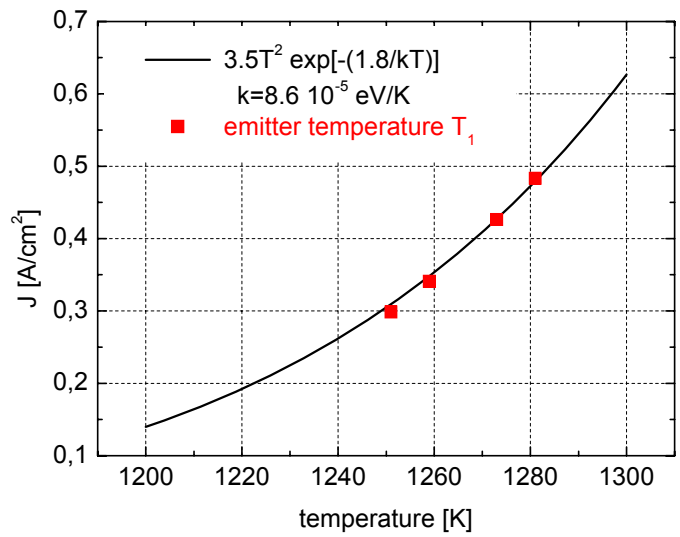

Fig. 10. Experimental emission data fitted by the RichardsonDushman law at the work function of $1.8 \mathrm{eV}$.

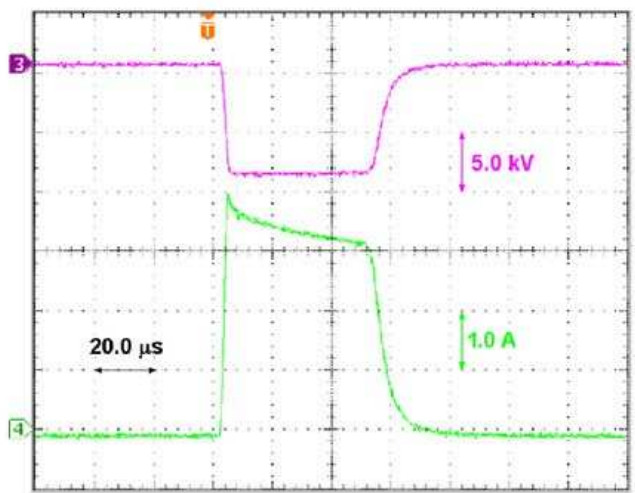

Fig. 11. Beam voltage (red trace) and emission current (green trace).

\section{CONCLUSION}

With emphasis on compactness and ease of use, much interest has been devoted to microwave generators that do not require an external magnetic field so as to reduce cost, size and weight of the device. Because of its inherent simplicity, one such device is the monotron, and for a $6.7 \mathrm{GHz}$ developmental device an electron gun has been constructed and tested.

Here described, the gun design has followed the basic requirement that the desired emission density should be obtained at a minimum cost. This requirement can be met with emitters that have a low work function and a moderate operating temperature. The power radiated by the cathode should obviously be as low as possible so that the heater power that compensates for losses by radiation and conduction can be kept low. Preliminary emission 
measurements have shown current densities of $0.5 \mathrm{~A} / \mathrm{cm}^{2}$ at around $1000{ }^{\circ} \mathrm{C}$. We conjecture that such a moderate performance stems from the poisonous effect of vaporized nickel coming from the cathode plate. To circumvent this problem, replacing the nickel disk (melting point $1400{ }^{\circ} \mathrm{C}$ ) by a one made of tantalum (melting point $2850{ }^{\circ} \mathrm{C}$ ) not only would restrain the presence of poisonous gases but also could make it possible to raise the operating temperature to the $1300-1400 \mathrm{~K}$ range with a consequent increase in the emission current.

\section{REFERENCES}

[1] J. J. Barroso, "Design facts in the axial monotron", IEEE Trans. Plasma Sci., vol. 28, pp. 652-656, June 2000.

[2] J.J. Barroso, J.O. Rossi, H. Patire Jr., P.J. Castro and J.A.N. Gonçalves, "A $25 \mathrm{kV} 10 \mathrm{~A}$ pulser for driving a high power Pierce electron gun", Proc. of the $7^{\text {th }}$ Brazilian Power Electronics Conference, pp. 63-64, Sept. 2003.

[3] J. A. N. Gonçalves, G. M. Sandonato, and C. M. Neto, "Characterization of a carbonate coating for oxide cathodes", Vacuum, vol. 49, no. 1, pp. 9-15, Jan. 1998.

[4] W. H. Kohl, Handbook of Materials and Techniques for Vacuum Devices, Woodbury, NY: AIP Press, 1995, ch. 16 